\title{
Author Correction: High-dimensional single-cell analysis predicts response to anti-PD-1 immunotherapy
}

Carsten Krieg (D, Malgorzata Nowicka, Silvia Guglietta, Sabrina Schindler, Felix J Hartmann (D), Lukas M Weber (D), Reinhard Dummer, Mark D Robinson (D), Mitchell P Levesque (D) and Burkhard Becher (D)

Correction to: Nature Medicine https://doi.org/10.1038/nm.4466, published online 8 January 2018.

In the version of this article initially published, Figs. 5a,c and 6a were incorrect because of an error in a metadata spreadsheet that led to the healthy donor patient 2 (HD2) samples being used twice in the analysis of baseline samples and in the analysis at 12 weeks of anti-PD-1 therapy, while HD3 samples had not been used. Data from sets of both samples should have been used in the analyses. The influence of this error on population proportions, marker expression, $P$ values and tSNE visualization (in Figs. 5a,c and 6a) was minimal (e.g., via clustering and variance calculations). Complete reanalysis of the dataset, now including data from both HD2 and HD3, resulted in minor changes to Figs. 5a,c and 6a as well as Supplementary Figs. 7, 9, 11 and 17. This error did not affect other analyses or any of the conclusions in the paper.

Also, there was an error in the description of the $n$ values in the original Fig. $6 \mathrm{~b}$ legend. The legend originally read: "(R and NR, $n=4$ for each group)". It should be: "(HD, $n=4 ; \mathrm{R}$ and $\mathrm{NR}, n=3$ for each group)". In addition, the Fig. 6 legend originally read: "All patients in this study were analyzed $(n=51)$ ". It should read: "All patients from the validation cohort were analyzed $(n=31)$, with any patient not making the 12-month endpoint (i.e., OS or follow-up) included in the 'No' column".

Additionally, there were errors in the Supplementary Information. In the 'Validation by flow cytometry' section of the Supplementary Methods, the antibody list was missing some antibodies. It originally read: "CD11b-BrilliantViolett (BV) 421 (ICRF44), CD14-PE (HCD14), HLA-DR-FITC (L243), CD4-BV711 (OKT4), CD33-BV605 (WM53), and Live/dead-stain-NearInfraRed.” This list should include CD19-BV605 (1D3) and CD3-BV785 (OKT3). In the "Correlation of PFS with monocyte frequency" section of the Supplementary Methods, there was an error in the sentence, "To compute the cumulative hazard function we used the previously calculated cutoff of $19.39 \%$ to create the 2 groups". The percentage was incorrect. It should be $19.38 \%$.

The errors have been corrected in the HTML and PDF versions of the article. 

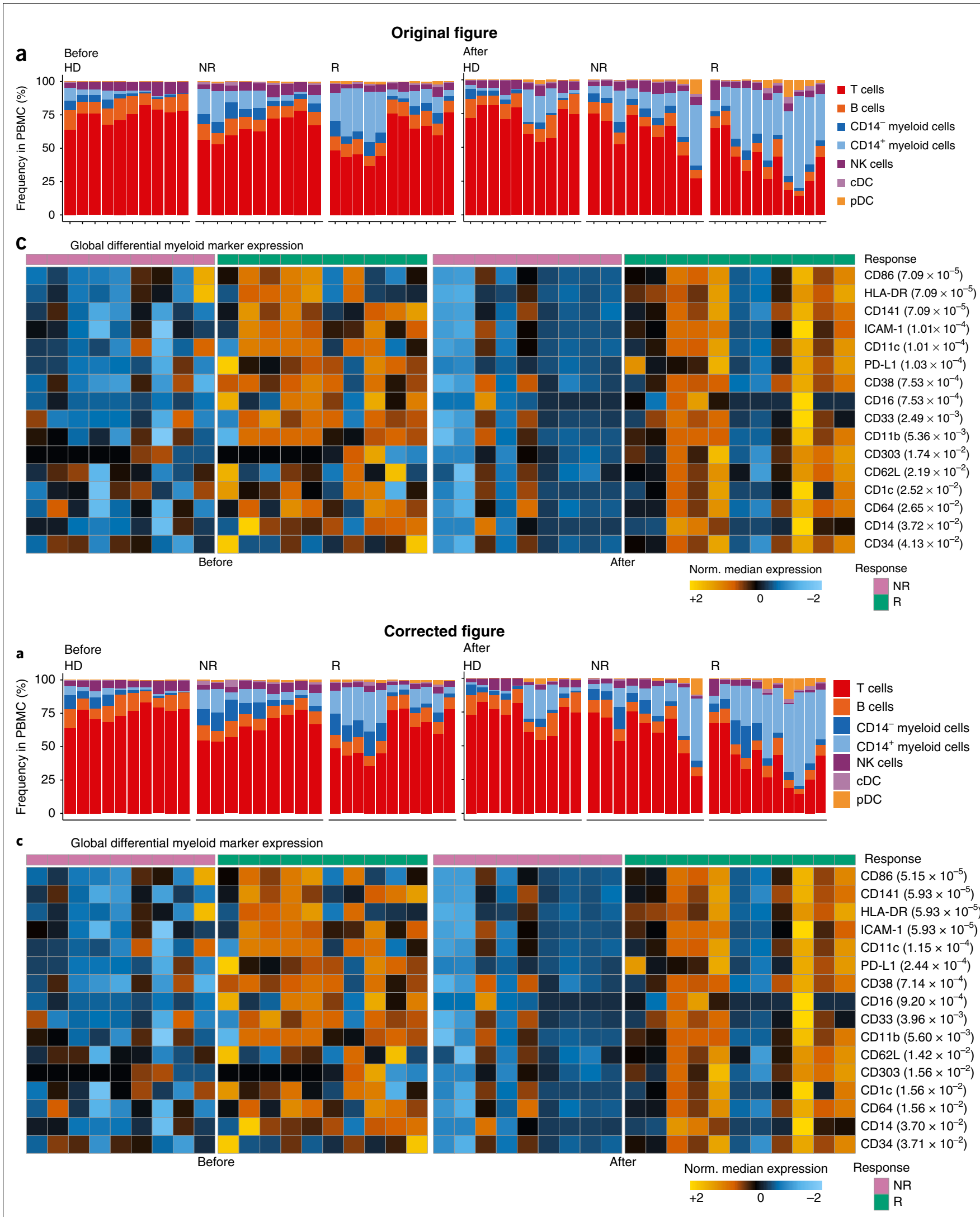

Fig. 5 | Orginal and Corrected. 


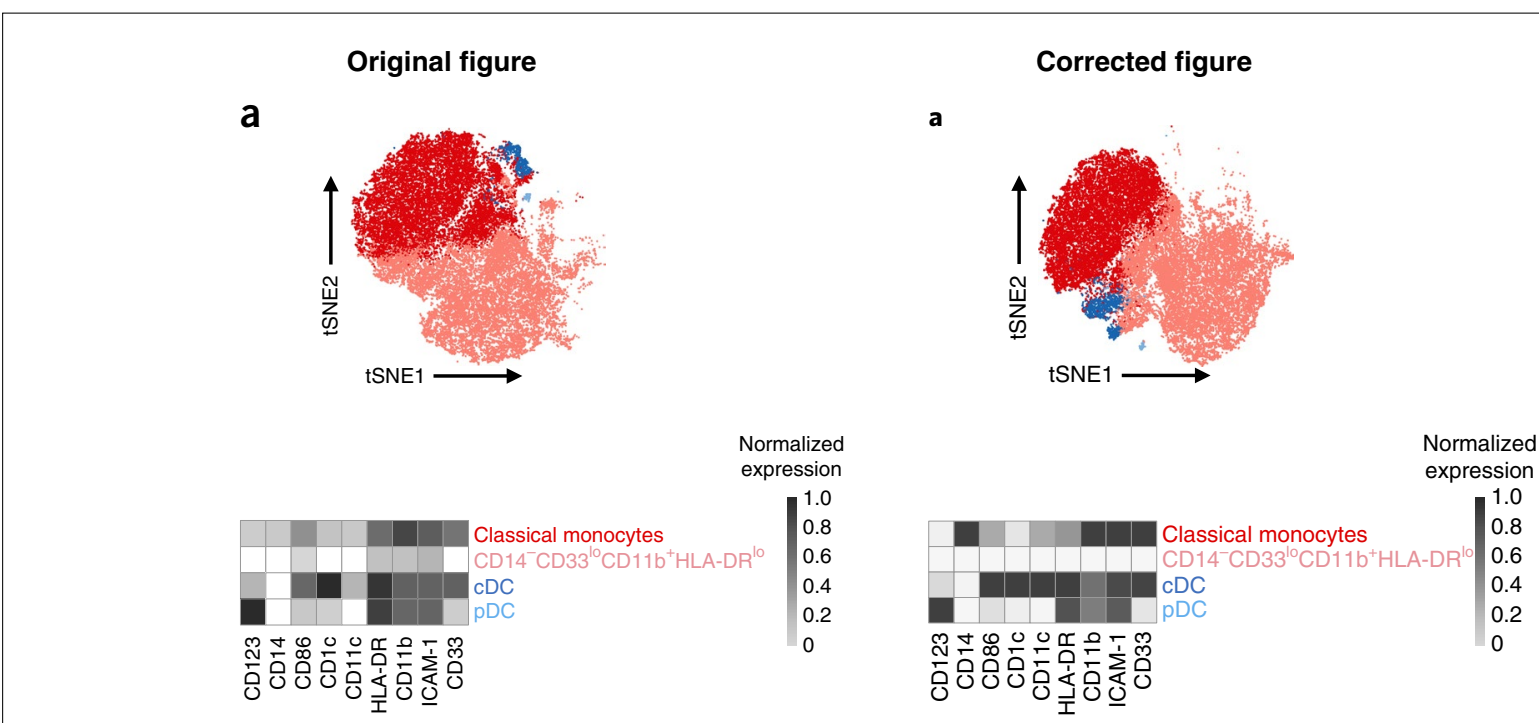

Fig. 6 | Orginal and Corrected. 